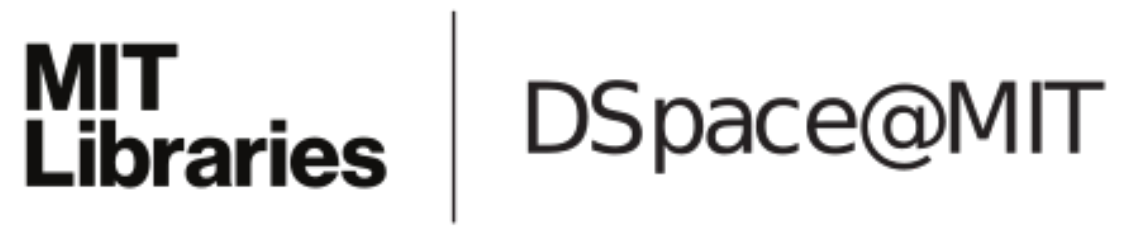

\author{
MIT Open Access Articles
}

\section{Device Prototype for Vaginal Delivery of Extremely Preterm Fetuses in the Breech Presentation}

The MIT Faculty has made this article openly available. Please share how this access benefits you. Your story matters.

Citation: Whalen, Mallory, Chang-Davidson, Elizabeth, Moran, Terra, Hoffman, Rachel, Frydman, Galit $\mathrm{H}$ et al. 2021. "Device Prototype for Vaginal Delivery of Extremely Preterm Fetuses in the Breech Presentation." Journal of Medical Devices, 15 (2).

As Published: 10.1115/1.4049086

Publisher: ASME International

Persistent URL: https://hdl.handle.net/1721.1/139670

Version: Final published version: final published article, as it appeared in a journal, conference proceedings, or other formally published context

Terms of Use: Article is made available in accordance with the publisher's policy and may be subject to US copyright law. Please refer to the publisher's site for terms of use. 
Mallory Whalen ${ }^{1}$

Department of Mechanical Engineering,

Massachusetts Institute of Technology,

Cambridge, MA 02139

e-mail: malw@mit.edu

Elizabeth Chang-Davidson

Department of Mechanical Engineering,

Carnegie Mellon University,

Pittsburgh, PA 15213:

Department of Mechanical Engineering

Massachusetts Institute of Technology,

Cambridge, MA 02139

e-mail: echangda@andrew.cmu.edu

Terra Moran

Master in Design Engineering Program,

Harvard University,

Cambridge, MA 02138

e-mail: moranterra@gmail.com

Rachel Hoffman

Department of Mechanical Engineering,

Massachusetts Institute of Technology,

Cambridge, MA 02139

e-mail: rachelmh@mit.edu

Galit H. Frydman

Department of Biological Engineering, Massachusetts Institute of Technology,

Cambridge, MA 02139;

Department of Surgery,

Massachusetts General Hospital,

Boston, MA 02114

e-mail: grrydman@mit.edu

Alexander Slocum

Professor

Fellow ASME

Department of Mechanical Engineering,

Massachusetts Institute of Technology,

Cambridge, MA 02139

e-mail: slocum@mit.edu

Alissa Dangel

TL1 Clinical Research Fellow,

Tufts Medical Center-CTSI,

Boston, MA 02111

e-mail: adangel@tuftsmedicalcenter.org

\section{Device Prototype for Vaginal Delivery of Extremely Preterm Fetuses in the Breech Presentation}

Vaginal delivery is typically avoided in the extremely preterm breech population due to the concern of entrapment by the cervix of the aftercoming head. A mechanical device concept is presented to enable vaginal delivery by preventing retraction of the cervix against the fetus during delivery. The two-part device was designed to dilate the cervix, prevent prolapse of small fetal parts and maintain sufficient dilation during delivery. The two-part device was designed and manufactured with the following modules: an inflatable saline-filled cervical balloon for dilation and a cervical retractor composed of semirigid beams to stabilize the cervix and maintain adequate dilation. The device was tested using a cervical phantom designed to simulate the compressive force the cervix exerts. The cervical balloon reached a maximum dilation of $8.5 \mathrm{~cm}$, after which there was leakage of saline from the balloon. While this dilation was less than the target goal of $10 \mathrm{~cm}$, the leaking was attributed to prototype manufacturing defects, which could be resolved with further development. The cervical retractor was able to withstand between 1-3 kPa. Although estimates of cervical pressure values can be upward of $30 \mathrm{kPa}$, there are no in vivo measurements to formally identify the pressure values for patients in preterm labor. This device serves as a viable proof-of-concept for utilizing an inflatable balloon device to prevent cervical retraction in the setting of extremely preterm vaginal breech delivery. Further manufacturing improvements and design changes could improve the device for continued development and testing. [DOI: 10.1115/1.4049086]

\section{Introduction}

In most pregnancies that are carried to term, the fetus has typically moved into a cephalic (head first) presentation prior to delivery. Breech presentation occurs when the fetus has not moved into a head-down position and the buttocks and abdomen are positioned to exit through the birth canal before the head. The probability of breech presentation decreases with advancing gestational age. The rate of breech presentation at term (greater than 37 weeks) is $3-4 \%$ [1]. In stark contrast to fetuses born at term, extremely preterm (less than 28 weeks) fetuses have a $25 \%$ likelihood of breech presentation [2].

\footnotetext{
${ }^{1}$ Corresponding author.

Manuscript received February 12, 2020; final manuscript received October 22, 2020; published online December 18, 2020. Assoc. Editor: Prasanna Hariharan.
}

Preterm fetuses exhibit a cone shape, where the head diameter is substantially larger than the lower body diameter, more so than in full term fetuses. In a vaginal delivery with breech presentation, the cervix may dilate only enough to allow the lower body to exit. This can then result in head entrapment by the insufficiently dilated cervix (Fig. 1). Head entrapment can cause poor oxygenation and blood flow in the fetus due to pressure around the fetus' neck or the umbilical cord. Due to this risk, doctors usually resort to delivering the fetus via Cesarean section (C-section) despite continued controversy over the benefits of cesarean delivery in this setting [3]. The type of C-section used at very early gestational age is associated with increased maternal morbidity, including the need for blood transfusions [4-6].

To enable vaginal delivery of extremely preterm fetuses, a device that both expands the cervix to $10 \mathrm{~cm}$ and holds it open to 


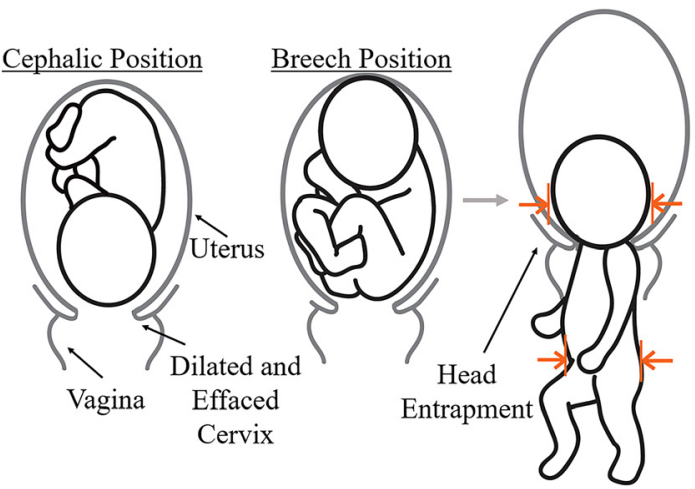

Fig. 1 Schematic of the cephalic position, breech position, and head entrapment with anatomy labeled

prevent head entrapment is needed [1]. To the authors' knowledge, there are currently no devices on the market that expand the cervix up to $10 \mathrm{~cm}$ for birth, nor any devices that are used for cervical retraction during delivery. However, there are several methods, such as osmotic expanders, rigid dilators, and balloons that are used to dilate the cervix up to $4 \mathrm{~cm}$ [7]. The precedent for a balloon type device to dilate the cervix is strong since Cook Cervical Ripening Balloons and Foley catheters are currently used to initiate cervical dilation [8,9]. Obstetric providers control dilation of balloon dilators with the volume of fluid pumped into the balloon [10]. Balloons used for cervical dilation are considered safe for the patient and easy for clinicians to use [11]. In the case of head entrapment, mechanical dilation of the cervix with a balloon based device would appear to be a viable and clinically acceptable option. To prevent head entrapment, our proposed solution is a two part device; a cervical balloon that dilates the cervix and a cervical retractor that holds the cervix and birth canal open during delivery (Fig. 2). As with any device in obstetrics that will be used in close proximity to the fetus, the device needs to be made of reasonably soft material to prevent direct injury to the fetus, such as bruising, nerve damage, or bone fracture. The cervical balloon is placed inside the cervical retractor and both devices, uninflated, are inserted into the cervix. The uterine ring is then advanced slightly and placed in the lower uterine segment. The perineal ring remains just outside of the vaginal introitus and rests against the perineum. The cervical dilation balloon is inflated slowly over a period of time, which may take several hours. In emergent situations, the balloon can be inflated in tens of minutes, however, the inflation rate should be determined by the clinician. The balloon inflation also facilitates the ring balloons unfolding. Once the cervix reaches optimal dilation, the two rings are inflated with saline and become rigid. The cervical dilation balloon is then deflated and removed, leaving an open channel through the cervical retractor for delivery. After delivery of the fetus, the rings are deflated and the device is removed.

\section{Materials and Methods}

2.1 Design Criteria. The need for this device was identified by Dr. Alissa Dangel, who also was a design team member and participated in its development and testing using a deterministic peer-review based method for rapid device development [12,13]. The design criteria are summarized in Table 1. The proposed device would ideally dilate the cervix from $4 \mathrm{~cm}$ to $10 \mathrm{~cm}$ (Fig. 2). In clinical practice, the precise dilation at which delivery is inevitable varies with the clinical situation. However, for purposes of device development, $4 \mathrm{~cm}$ of cervical dilation was the point chosen as the minimum dilation at which the device would be placed [14]. Medical consensus is that $10 \mathrm{~cm}$ is the necessary dilation diameter for delivery of a fetus [1]. However, dilation slightly below $10 \mathrm{~cm}$ may still be acceptable as the device is intended for extremely preterm fetuses and the 95th percentile for a biparietal diameter of a male fetus at 28 weeks is $8.04 \mathrm{~cm} \mathrm{[15].}$

With regard to cervical geometry, the primary variables used by clinicians are dilation and effacement. The cervix is approximately a hollow cylinder in shape, with effacement corresponding to the height of the cylinder and dilation corresponding to the diameter of the internal os. For the cervical model, the starting shape, at time zero, is assumed to be $4 \mathrm{~cm}$ of dilation and $50 \%$ $(2 \mathrm{~cm} / 4 \mathrm{~cm})$ effaced. The shape at the final time $(f)$ is $10 \mathrm{~cm}$ of dilation and approximately $100 \%$ effaced $(0.1 \mathrm{~cm} / 4 \mathrm{~cm})$ as described in Eqs. (2) and (3) [14].

Prior research was consulted to estimate the force required to dilate the cervix. Research has been done on mechanical properties of the cervix in vitro at term and early term [16]. There also exists some in vivo data of cervical forces during pregnancy, but limited data exists on cervical properties and forces at the time of birth [17]. Measuring these properties is not easy, nor of priority during delivery. Therefore, assumptions about forces must be made from existing data to model the scenario of delivery at a gestational age of around 28 weeks.

Using data from in and ex vivo testing of pregnant cervixes, the pressure needed to dilate the cervix was overestimated to be about $30 \mathrm{kPa}[18-20]$.

The area of the cervix is approximated as the outer surface of a cylinder

$$
\begin{gathered}
A=2 \pi r h \\
\text { At } t=0, A=2 \pi(2 \mathrm{~cm})(2 \mathrm{~cm})=8 \pi \mathrm{cm}^{2} \\
\text { At } t=f, A=2 \pi(5 \mathrm{~cm})(0.1 \mathrm{~cm})=\pi \mathrm{cm}^{2}
\end{gathered}
$$

When determining the maximum anticipated pressure, the minimum area is $A=\pi \mathrm{cm}^{2}$. From Drunecký et al., force is estimated to be $10 \mathrm{~N}$

$$
P=\frac{F}{A}=\frac{10 \mathrm{~N}}{\pi \mathrm{cm}^{2}} \approx 30 \mathrm{kPa}
$$

This matches Hee et al., where $P=4-15 \mathrm{kPa}$, with a safety factor of 2 .

This pressure was chosen to ensure that the device could dilate a cervix. The cervical pressure was assumed to be constant and time-related mechanical properties were ignored. In clinical use, doctors can control the inflation of the device and can account for viscoelastic or other time dependent mechanical responses with feedback from the patient and/or clinical exam information.
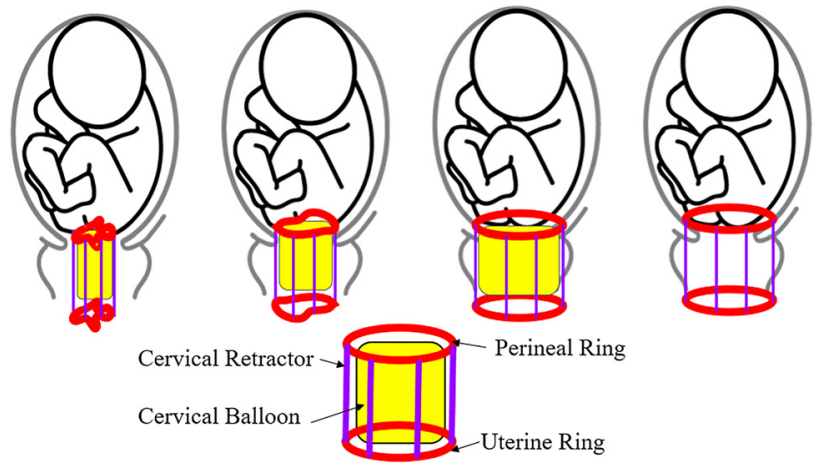

Fig. 2 Schematic of the two part device to dilate cervix and hold birth canal open. A cervical balloon dilates the cervix while a cervical retractor device with a ring balloon (uterine ring) in the uterus and a ring balloon (perineal ring) outside the vagina connected together with beams holds the birth canal open. 
Table 1 Table of design criteria

\begin{tabular}{lll}
\hline \hline Design criteria & & \\
\hline Requirements & Cervical retractor & Cervical balloon \\
Safely deliver fetus & $\bullet$ Anchor in uterus & $\bullet$ Dilate cervix to $10 \mathrm{~cm}$ \\
& $\bullet$ Keep birth canal open & $\bullet$ Block birth canal until delivery \\
& $\bullet$ No sharp edges & $\bullet$ Expand radially and not axially \\
& $\bullet$ Take up minimal space in uterus & $\bullet$ Slow, controlled inflation \\
Minimal harm to mother & $\bullet$ Remains open for minimal time & $\bullet$ Expand under constant pressure of 30 kPa \\
& $\bullet$ No sharp edges & \\
Withstand forces of birth & $\bullet$ Rings must remain open & $\bullet$ Use sanitizable materials \\
Medically compatible & $\bullet$ Beams must not deflect $>1 \mathrm{~cm}$ into canal & $\bullet$ Easy for physician to use \\
\hline \hline
\end{tabular}

The device must also hold the cervix open during delivery. Although some research has been completed on cervical material properties, the viscoelasticity of cervical tissue at the time of delivery is not well understood [16,17]. Therefore, there is little evidence to suggest how long the cervix will maintain its dilation and there is a risk that the cervix could retract prior to delivery being completed, thus leading to head entrapment. A device that holds the cervix open during delivery will reduce this risk.

Aside from forces directly associated with the cervix, there are additional forces that occur during birth. Forces from contractions and the pelvic floor muscles were not considered in this phase of development. The fetus can also exert a downward force on the cervix. Research by Allman et al. reported this force to have a value of $0.8 \mathrm{~N}$ and that was used as an additional parameter for the device design [21].

2.2 Cervix Phantom Development. A cervix phantom was developed for device testing (Fig. 3). This provides a necessary first benchmark to ensure that the device can overcome the pressure exerted by a cervix during forced dilation. No cervical models that mimic pressure, effacement, and dilation of the cervix are known by the authors to exist at this time. Additionally, definitive values for pressure needed to dilate the cervix up to $10 \mathrm{~cm}$ are not known, so the calculated pressure of $30 \mathrm{kPa}$ was used to design the cervix phantom.

The design involved a loop of material placed in tension by a high extension spring. To determine the correct spring constant, the force of the spring was related to pressure and balloon size according to Eq. 8. As shown in Fig. 4, $r$ is balloon radius, $x$ is

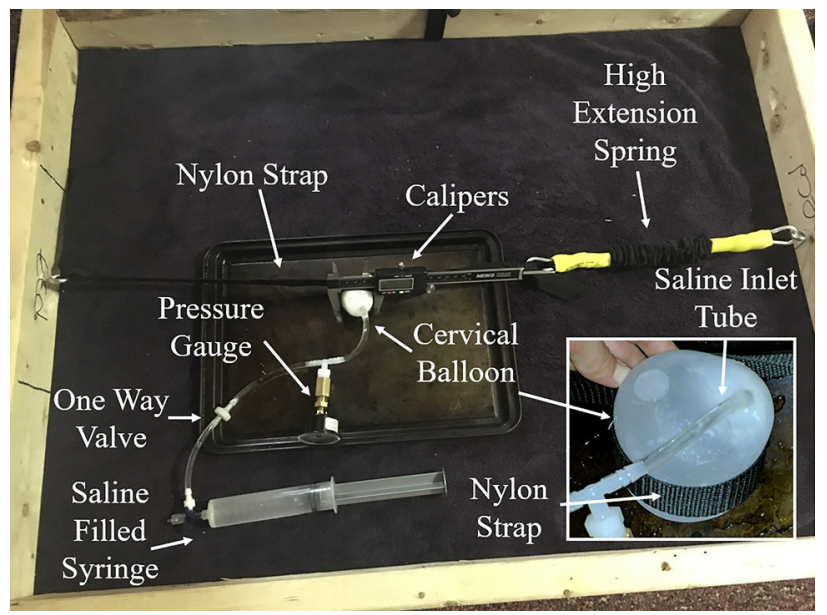

Fig. 3 Cervix phantom comprising of a wooden frame, eyelet screws, nylon webbing, and high extension spring. Close up of cervical balloon being tested in the phantom on bottom right. length of spring extension, $h$ is strap height, $T$ is the tension in the band, and $P$ is the internal balloon pressure

$$
\begin{gathered}
2 T=2 P r h=2 P\left(r_{0}+\Delta r\right) h \\
T=k x=k\left(x_{0}+2 \pi \Delta r\right) \\
T=P\left(r_{0}+\Delta r\right) h=k\left(x_{0}+2 \pi \Delta r\right) \\
k=\frac{P\left(r_{0}+\Delta r\right) h}{x_{0}+2 \pi \Delta r}
\end{gathered}
$$

To expand under a constant $30 \mathrm{kPa}$ of pressure, the spring needs to be nonlinear. However, it is unclear if the cervix resists dilation with constant pressure so this requirement may not be strictly necessary. A spring constant of $131 \mathrm{~N} / \mathrm{m}$ was chosen to test the device.

2.3 Cervical Retractor Development. A cervical retractor component was designed to maintain cervical dilation during delivery. It was constructed of two inflatable rings: one placed in the lower uterine segment (uterine ring) and the other placed outside of the vagina on the perineum (perineal ring). Six stabilizing beams were used to connect the rings and provide rigidity to counter the pressure by the cervix and birth canal. Final dimensions of the retractor were as follows: maximal outer diameter of $14 \mathrm{~cm}$, inner diameter of $12 \mathrm{~cm}$, and a cross-sectional diameter of $1 \mathrm{~cm}$.

In this prototype, the perineal and uterine rings are identical. The device cannot be placed backward as the beams are symmetric. In future iterations, the perineal ring may differ from the uterine ring to provide greater stiffness.

The beams are designed to follow the shape of the pelvis, modeled from the pelvic curvature of standard forceps and thus they are curved (Fig. 5). The beams have a "T" cross section to allow their ends to fit into molded features in the inflatable rings and not twist as the uterine rings inflate. The " $T$ " also provides sufficient rigidity and bending strength. The flat surface of the " $T$ " faces the open channel to ensure nothing protrudes into the passageway while the fetus is delivered. The exterior surface of the columns
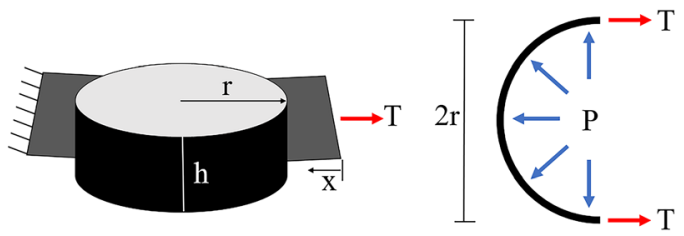

Fig. 4 (Left) Diagram of cervix in phantom for Eq. (5). (Right) Free body diagram of the phantom strap around the cervix balloon. 

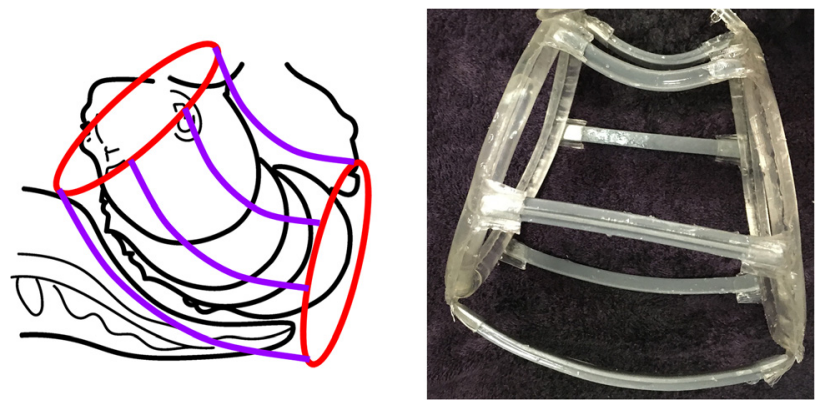

Fig. 5 Redesign of cervical retractor beams to follow the curvature of the pelvis

could also be wrapped in a plastic sheeting to ensure the sections of the cervix not supported by the relatively thin columns are also held open sufficiently.

The number of beams in this prototype was set at six. This was an attempt to sufficiently distribute the load over the individual beams, while balancing the need to maximize available space. Fewer beams would result in a reduction of the available space for a fetus to pass through due to the inability to create a circular shape. However, an excessive number of beams would become cumbersome and difficult to insert into the cervix at $4 \mathrm{~cm}$ dilation.

High-density polyethylene (HDPE) was selected for its elastic modulus to provide sufficient rigidity to prevent bending and subsequent protrusion of the columns into the birth canal under load. The prototype devices uses $3 \mathrm{D}$ printed beams to enable tests to rapidly converge on the final geometry, but injection moldable HDPE or similar biocompatible plastics should be used in any future prototypes that might be tested in patients.

The beam performance was predicted through initial closed form analysis verified with a finite element model to check for buckling of a circular arch under continuous load. Each model had margin for error, so both methods were checked against each other to help rapidly evolve the design and enable future scaling of the device. The software ABAQUS standard (version R2017x), was used for the finite element model. The mesh used linear hexahedral elements of type C3D8R with an approximate global size of 1 (Fig. 6). The mesh was dependent on the beam geometry. The beams were modeled as pinned on both ends. The cervix is approximated as a cylinder with a $30 \mathrm{~cm}$ circumference and a $1 \mathrm{~cm}$ height that exerts a pressure of $30 \mathrm{kPa}$ over its entire area. Thus, a total force of $94 \mathrm{~N}$ is applied to the six columns of the cervical retractor. Assuming the load is distributed equally over the six columns means that each column experiences a $15.7 \mathrm{~N}$ load. Dividing this force over the area of contact for each column $\left(1^{2} \mathrm{~cm}\right)$ equates to a pressure of $157 \mathrm{kPa}\left(0.157 \mathrm{~N} / \mathrm{mm}^{2}\right)$. In

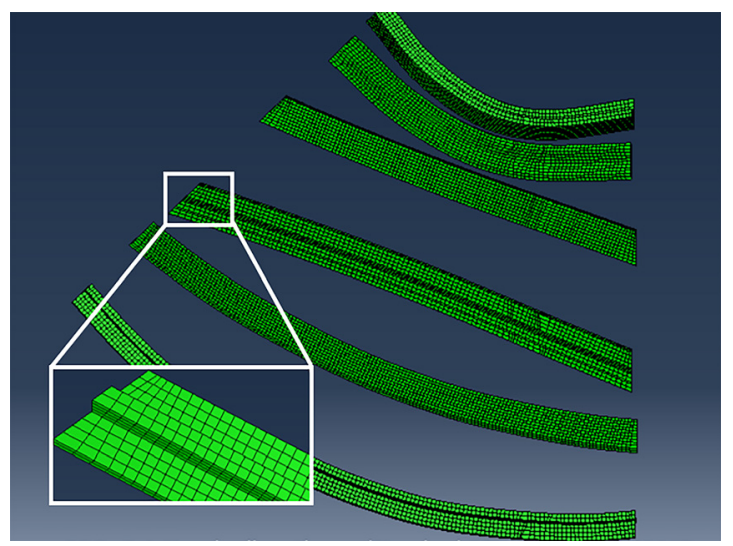

Fig. 6 ABAQUS model of the cervical retractor beams with the mesh shown addition to the pressure applied by the cervix, an additional pressure of $5 \mathrm{kPa}\left(0.005 \mathrm{~N} / \mathrm{mm}^{2}\right)$ was applied to the outer surface of the columns to represent the pressure imposed by the birth canal (Fig. 7).

The finite element model resulted in a maximum stress of $1.04 \times 10^{7} \mathrm{~N} / \mathrm{m}^{2}$, which is less than the yield strength of HDPE, around $2.7 \times 10^{7} \mathrm{~N} / \mathrm{m}^{2}$. The maximum stress occurs at the end connection of the columns, which is an area of error for the model. The maximum stress along the length of the column is $4.23 \times 10^{6}$ $\mathrm{N} / \mathrm{m}^{2}$. The maximum deflection, $3 \mathrm{~mm}$, is at the location of the load from the cervix (Fig. 8).

Analysis estimated a deflection of up to $5.25 \mathrm{~mm}$ and a critical buckling load over $84 \mathrm{~N}$. For analysis, deflection of a fixed end flat beam with a uniform load was used (Eq. (9)). Since the beams are curved and materials fail in tension, the equation overestimates the deflection for the convex beams and underestimates it for the others

$$
\delta_{\max }=\frac{w l^{4}}{384 \mathrm{EI}}
$$

The formula for critical pressure at which buckling of a uniformly compressed circular arch occurs (Eq. 10) was used [22]

$$
q_{\text {crit }}=\frac{\mathrm{EI}}{\bar{R}^{3}}\left(\frac{\pi^{2}}{\alpha^{2}}-1\right)
$$

Although the analysis methods are not in perfect agreement, they provided the confidence to move forward with manufacturing and testing. Even in the most extreme predicted case, there will be sufficient space for the fetus to be delivered through the cervical retractor $(<1 \mathrm{~cm}$ deflection) and the beams should not fail under load. The uterine and perineal rings were thermoformed from flexible thermoplastic polyurethane sheeting. This material was selected to allow for sufficient malleability so that the rings can be folded during insertion. The beams are attached using thermoformed pockets on the rings. When the uterine rings are inflated they become rigid and expand to the desired diameter of $12 \mathrm{~cm}$, ideally large enough to not be expelled by the uterus during delivery. Ring rigidity is imperative to ensure the cervix stays open during delivery. Rigidity of the rings was estimated using calculations that predict critical buckling pressure of a pressurized toroidal ring [23]. This calculation considers several variables, including the ring wall thickness and internal pressure. The material used for prototyping had a wall thickness of $0.16 \mathrm{~mm}$, which equates to a critical buckling pressure of $1 \mathrm{kPa}$ with an internal

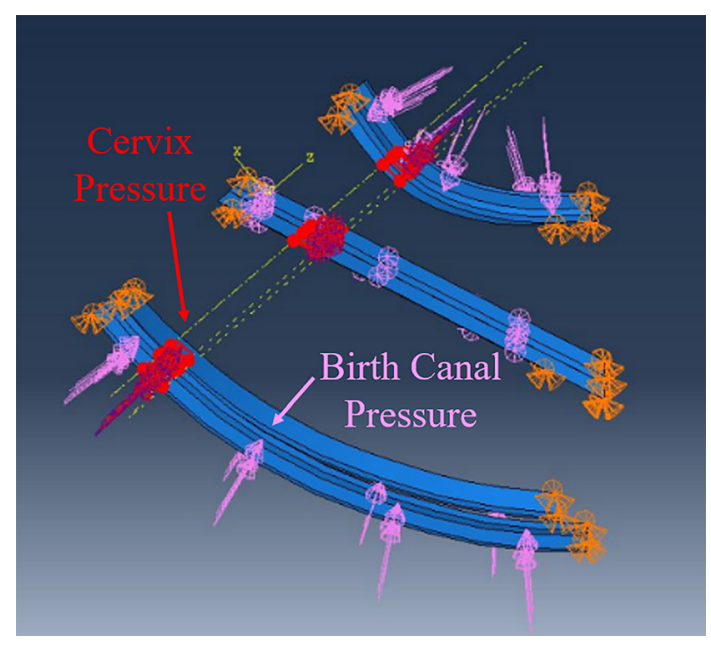

Fig. 7 ABAQus model of cervical retractor with locations of the pressure experienced while holding open the cervix and birth canal 


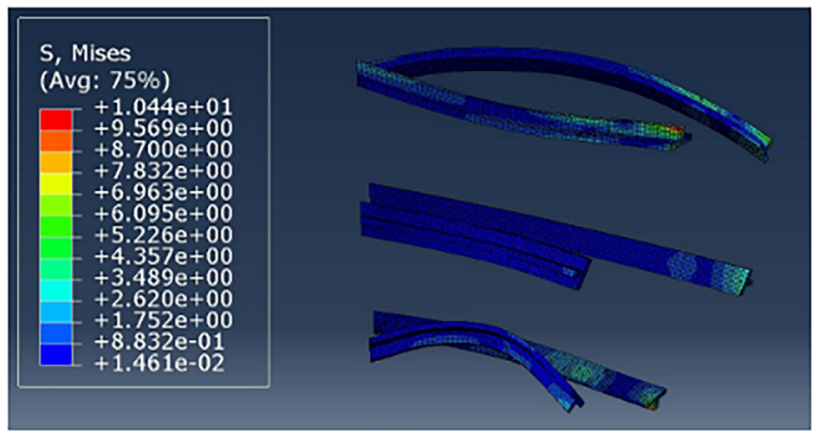

Fig. 8 Von Mises stress and deformations in the finite element model. Stress is displayed in $\mathrm{N} / \mathrm{mm}^{2}$.

pressure of $1 \mathrm{kPa}$. This closely matches the testing result of $1-3 \mathrm{kPa}$ buckling pressure. The following equations (Eqs. (11)-(13)) determine $P_{b}$, the out of plane ring buckling circumferential pressure given the constants in Eqs. (14)-(21):

$\overline{q_{\mathrm{cr}}}=\frac{9+\frac{1.125 \Gamma}{P+S}}{4+5 c+\frac{1+5 c}{\Gamma}+\frac{12.375+13.875 c}{S+P}+\frac{\Gamma}{P+S}(0.5+0.625 c)}$

$$
\begin{gathered}
q=\frac{\overline{q_{\mathrm{cr}}} \mathrm{EI}}{R^{3}} \\
P_{b}=\frac{q}{2 \pi R}
\end{gathered}
$$

Given the following constants:

$$
\begin{gathered}
\Gamma=\frac{J G}{\mathrm{EI}} \\
S=\frac{A G R^{2}}{2 \mathrm{EI}} \\
P=\frac{p \bar{A} R^{2}}{\mathrm{EI}} \\
A=2 \pi r t \\
\bar{A}=\pi r^{2} \\
c=\frac{r}{R} \\
I=\pi r^{3} t \\
J=2 \pi r^{3} t
\end{gathered}
$$

Calculations indicate that a completely solid ring of thermoplastic polyurethane would be needed to withstand $30 \mathrm{kPa}$. However, a slightly stiffer material could be used in an inflatable device to resist buckling under higher pressure values. Preliminary testing was done to confirm that thicker walls would improve the device with regards to the point at which buckling would occur. A test retractor with rings made from polyurethane tubing, outer diameter $12.7 \mathrm{~mm}(0.5 \mathrm{in})$ and inner diameter $6.35 \mathrm{~mm}$ (0.25 in), demonstrated the ability to withstand approximately $30 \mathrm{kPa}$ of pressure applied by the cervical phantom. The tubing was not internally pressurized and was connected to itself using a 3D printed doubleended tube stopper that defined the ring's diameter through it's own curvature. However, it is expected that the pressure required to maintain cervical dilation at $10 \mathrm{~cm}$ will be significantly less than $30 \mathrm{kPa}$ estimated for this study, as the cervix will have already dilated to the full diameter at this time. Knowing the cervical properties at the point of full dilation would help clarify the maximum pressure values exerted and guide subsequent design improvements of the uterine and perineal rings.

2.4 Cervical Balloon Development. A balloon concept was selected to dilate the cervix, as saline-filled balloons are common in medical applications [24]. Initially, both compliant (elastic) and noncompliant balloon materials were considered, but a compliant design was selected due to nonlinear effects in noncompliant balloons. To achieve the desired expansion from $4 \mathrm{~cm}$ to $12 \mathrm{~cm}$ in diameter, a compliant balloon material needs to accommodate a stretch of at least $300 \%$. A two part silicone platinum rubber was selected to provide the necessary stretch and to represent a medically compatible material. A wall thickness of $0.15 \mathrm{~cm}$ was selected for initial testing, as that would be thin enough to inflate by hand, but thick enough to mold easily. A higher wall thickness also decreases the effect of effacement in concentrating force on the balloon. As wall thickness increases, the assumption of low internal resistance to bending becomes less valid. The bending resistance of the balloon will reduce the deformation, distributing the load across more of the surface of the balloon. Another measure to decrease the force concentrations caused by effacement is to reduce expansion in the vertical direction. This also reduces the risk of the balloon protruding into the uterus. A central column was molded in the balloon to resist excessive vertical expansion as well as to decrease the amount of saline needed to inflate the device. ABAQUS was used to verify that the central column would create the desired balloon expansion (Fig. 9). The simulation accounted for nonlinear behavior by using the Ogden hyperelastic material model. Additionally, effectively inextensible Kevlar strings were placed vertically along the outside edge to reduce excessive vertical expansion. Processes for incorporating the Kevlar strings included direct application with glue or incorporation into the wax mold. Once the geometry of the balloon was finalized, lost wax casting with a $3 \mathrm{D}$ printed mold was used to create the balloons (Fig. 10).

2.5 Syringe-Pump System. The system for inflating the device consists of commonly available medical syringes, intravenous (IV) saline bags, and tubing connectors so that medical practitioners can easily operate the device. The system employs three-way stopcocks that connecting the IV bag, balloons, and $60 \mathrm{~mL}$ syringes. The syringes are used to pump saline out of the bag and into the balloon. For the cervical balloon, a one way valve is used as well as an additional syringe to deflate the balloon (Fig. 11). The amount of force necessary to operate the system

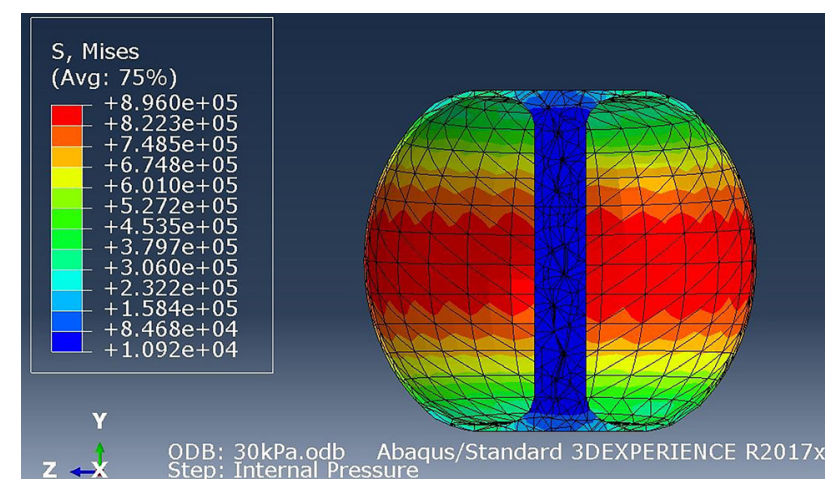

Fig. 9 ABAQUS simulation showing von Mises stress and radial expansion of the balloon when axial expansion is constrained with a central column. The simulation used an internal pressure of $30 \mathrm{kPa}$. Stress is displayed in $\mathrm{N} / \mathrm{mm}^{2}$. The balloon stretches radially because it experiences the most stress on the outer surface. 

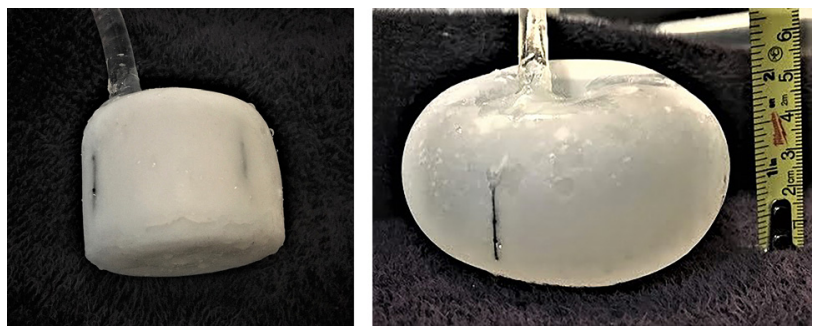

Fig. 10 Cervical balloon pre-inflation, left, and postinflation, right, showing preferential inflation in the radial direction. Initial height is $3 \mathrm{~cm}$ and initial diameter is $4 \mathrm{~cm}$. Final height is $5 \mathrm{~cm}$ and final diameter is $8.5 \mathrm{~cm}$.
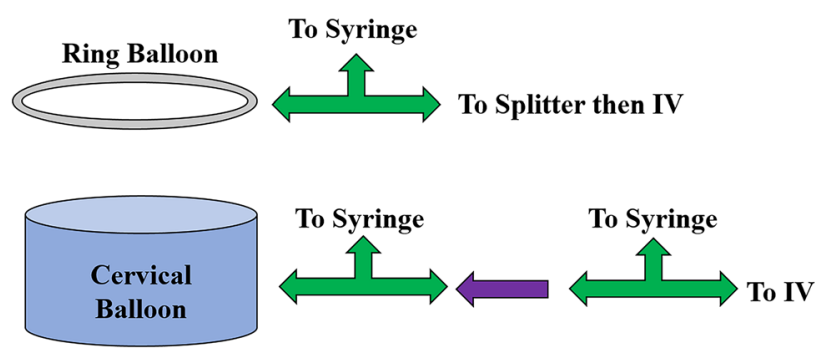

Fig. 11 Schematic of syringe pump system. The three headed arrows represent three-way stop cocks. The single headed arrow represents a one-way valve.

manually was calculated to ensure that the balloon could be filled by healthy practitioners. The force a practitioner's thumb would need to exert on the syringe was found to be $20 \mathrm{~N}$. Through experimentation, this was found to be within the range of operational comfort.

\section{Results}

3.1 Cervix Balloon Testing. Several iterations of the cervical balloon were tested using the cervix phantom. The maximum dilation achieved by a balloon in the cervix phantom was $8.5 \mathrm{~cm}$ of dilation. The reason these trials could not progress beyond this level of dilation was due to manufacturing defects of the balloon that resulted in leakage at that point. Our resources to manufacture the balloons were limited, but the technology sound, so achieving larger more reliable rings this should not be an issue for appropriately funded further work. The phantom was manually tested by a board-certified obstetrician-gynecologist with experience in cervical exams to confirm that the stiffness of the model was consistent with the expected in vivo level of cervical stiffness during labor. During testing, the balloon produced maximum pressure values of about $30 \mathrm{kPa}$, which aligns well with our estimate of $30 \mathrm{kPa}$ necessary to dilate the cervix. An in line dial pressure gage, $\pm 2 \%$

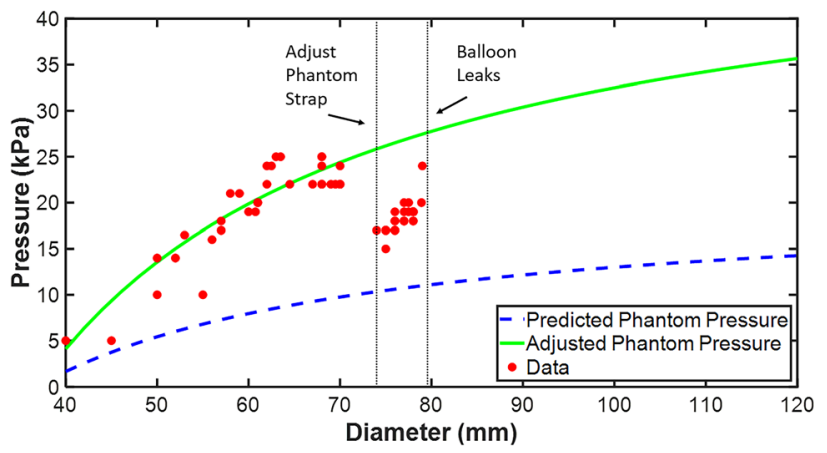

Fig. 12 Testing results and analysis for cervical balloon inflation accuracy, and $2 \mathrm{kPa}$ resolution, measured the pressure in the balloon. A one-way valve prevented back flow and allowed for a static pressure measurement. Additionally, the pressure values recorded are similar to predicted pressure values, with a constant factor of 2.5 between them. Measured pressure in the balloon, predicted pressure the phantom applies, and phantom pressure adjusted for friction are graphed versus the balloon's diameter (Fig. 12). The source of the difference between predicted and measured pressure is suspected to be due to friction, as there are certainly frictional forces between the balloon and retractor elements involved that were not accounted for in our spring-pressure relation. Assuming the difference is attributable to friction, this would correspond to a frictional constant of 0.15 , which is plausible for the materials we used. Future work would aim to reduce the coefficient of friction by about half by using biocompatible lubricants.

3.2 Cervical Retractor Testing. The cervical retractor was tested in a similar setup with a spring scale to determine the circumferential pressure that would lead to buckling of the inflated rings. The spring scale was used in place of a spring due to lack of availability of weak springs with known spring constants. Using the same relation, this showed that the retractor rings would buckle at $1-3 \mathrm{kPa}$ of pressure. This matches well the $1 \mathrm{kPa}$ calculated using an out of plane ring buckling hand calculation [23].

\section{Discussion}

4.1 Cervical Balloon. The maximum dilation of the cervix phantom achieved by the cervical balloon was $8.5 \mathrm{~cm}$, above the minimum necessary diameter of $8.04 \mathrm{~cm}$, but below the goal of $10 \mathrm{~cm}$. Improvements in the manufacturing quality of the balloon should increase the maximum dilation achievable due to the limiting factor of balloon expansion appearing to be a manufacturing defect and not an essential design failure. The manufacturing process typically inadvertently produced up to six holes in the balloon, along the junction between the tubing and the balloon, all of which were potential failure points resulting in leakage, in addition to the defects from inadequate liquid polymer degassing during the molding process. The connection between the tubing and the balloon may also be a potential source of leaks. This could be resolved by adding a shaped connection to reduce the concentration of stress. During testing, several balloons did not leak at this junction; therefore, it seems plausible that the silicone glue used would be acceptable if an appropriate application process was developed and tested.

4.2 Cervical Retractor. The measured $1-3 \mathrm{kPa}$ buckling pressure of the cervical retractor rings is well below the estimated target of $30 \mathrm{kPa}$ necessary to dilate the cervix, but calculations showed that increasing wall thickness could raise the buckling pressure significantly. However, when the cervix is fully dilated, the mechanical properties of the cervix would be expected to change and exert less pressure but to what degree is unknown. Determination of a more exact estimate of this pressure would be an important future step for research into devices for cervical retraction. Additionally, since the perineal ring never enters the body, it is possible to modify the design of the retractor to incorporate a solid perineal ring. In this case, it would likely be necessary for the clinicians to attach the perineal ring to the rest of the retractor after the cervix has been dilated since the solid ring would not be able to be folded and placed at the time of insertion.

4.3 Future Work. The initial device developed and tested can be greatly improved with advanced manufacturing techniques and technology, as well as a few design changes. However, the lack of available data on the material properties of the cervix, uterus, and vagina limit the extent to which the device can be evolved. Accordingly, the device itself could be modified in order 
to remedy the lack of data available about cervical properties at the time of birth. Attaching a digital pressure gage in line with the cervical balloon capable of logging data could collect valuable information on cervical properties that could then help to evolve the device. Similarly, a device that measures the volume of the balloon in concert with balloon pressure would provide additional information on the mechanics of cervical dilation. Because the device would eventually be used in a medical context, subsequent versions should be made with medical grade sterilizable materials. Additionally, the folded geometry of the device (including the cervical balloon, retractor, and tubing) will need to fit into the anticipated $4 \mathrm{~cm}$ diameter of a partially dilated cervix. Special packaging or an additional insertion device could be made to facilitate insertion. In future design iterations, the cervical retractor design should also seek to increase the pressure with standable by increasing uterine and perineal ring wall thicknesses or considering a solid perineal ring. If increasing the wall thickness on the uterine ring significantly decreases folding capabilities, linkages or hinges might be used but pinch points are of concern. Cervical balloon design changes should focus on increasing maximum dilation diameter as well as potentially adding features to encourage proper placement relative to the cervix. For the cervical balloon, more advanced manufacturing techniques will be needed to remove air bubbles, create even wall thicknesses, and patch holes inherent to the manufacturing process. There are many custom medical balloon sources and it is suggested to work with one of them for further balloon development. To counter the risk of balloon failure, a double-walled design or multiple balloons inside a single encasing could be used. In addition, in the case of a clinical emergency where quick removal is necessary, the balloons could potentially be attached to a suction system so that the saline could be removed rapidly and the device withdrawn quickly.

\section{Conclusions}

Development of this device prototype has shown that a balloon derived device can expand to $8.04 \mathrm{~cm}$ in the setting of a phantom used to mimic the cervix. This dilation can then be maintained with a retractor to create a channel through which the fetus can be delivered. Further development of this device could help obtain data on cervix' mechanical properties, in turn enabling development of better medical devices that engage the cervix. Ultimately, it is envisioned that an approved medical device will be developed that mitigates the risk of head entrapment by the cervix in the extremely preterm vaginal breech fetus to enable avoidance of $\mathrm{C}$ sections in this population.

\section{Acknowledgment}

We would like to thank Dr. Nevan Hanumara, Dr. Simona Socrate, Professor Ellen Roche, and Mary Caulfield from MIT and Dr. Michael House (Tufts Medical Center, Dept. Maternal Fetal Medicine) and Dr. Kumaran Kolandaivelu (MIT, Clinical Research Center) for their invaluable guidance and personal wealth of knowledge. We would also like to thank Nikayah Etienne.

The content is solely the responsibility of the authors and does not necessarily represent the official views of the NIH.

\section{Funding Data}

- National Center for Advancing Translational Sciences, National Institutes of Health (Award No. TL1TR002546; Funder ID: 10.13039/100006108).

\section{References}

[1] Yeomans, E. R., Hoffman, B. L., and Gilstrap, L. C., III, and Cunningham, F. G. 2017, Cunningham and Gilstrap's Operative Obstetrics, 3rd ed., McGraw-Hill Education LLC, New York.

[2] Gasim, T. G., 2008, "Preterm Singleton Breech Delivery in a Teaching Hospital of Saudi Arabia: Vaginal Versus Cesarean Delivery," J. Family Commun. Med., 15(2), pp. 65-70.

[3] Caughey, A. B., Cahill, A. G., Guise, J-M., and Rouse, D. J., 2014, "Safe Prevention of the Primary Cesarean Delivery," Am. J. Obstet. Gynecol., 210(3), pp. 179-193.

[4] Patterson, L., O’Connell, C., and Baskett, T., 2002, "Maternal and Perinatal Morbidity Associated With Classic and Inverted T Cesarean Incisions," Obstet. Gynecol., 100(4), pp. 633-637.

[5] Kawakita, T., Reddy, U., Grantz, K., Landy, H., Desale, S., and Iqbal, S., 2017, "Maternal Outcomes Associated With Early Preterm Cesarean Delivery," Am. J. Obstet. Gynecol., 216(3), pp. 312.e1-312.e9.

[6] Luthra, G., Gawade, P., Starikov, R., and Markenson, G., 2013, "Uterine Incision-to-Delivery Interval and Perinatal Outcomes in Transverse Versus Vertical Incisions in Preterm Cesarean Deliveries," J Matern. Fetal Neonatal Med., 26(18), pp. 1788-1791.

[7] Paul, M., Lichtenberg, E., Borgatta, L., Grimes, D., Stubblefield, P., and Creinin, M., 2009, Management of Unintended and Abnormal Pregnancy: Comprehensive Abortion Care, Wiley-Blackwell, Hoboken, NJ.

[8] Health Products for You, 2020, "Foley Catheters," HPFY, Brookfield CT, accessed Nov. 23, 2020, https://www.healthproductsforyou.com/c-foley-catheters. html

[9] Cook Medical LLC, 2020, "Cook ${ }^{\circledR}$ Cervical Ripening Balloon With Stylet," Cook Medical LLC, Bloomington, IN, accessed Nov. 23, 2020, https:// www.cookmedical.com/products/wh_crbs_webds/

[10] Abeyaratne, R., 1987, Lecture Notes on The Mechanics of Elastic Solids, Department of Mechanical Engineering, Massachusetts Institute of Technology, Cambridge, MA.

[11] Gu, N., Ru, T., Wang, Z., Dai, Y., Zheng, M., Xu, B., and Hu, Y., 2015, "Foley Catheter for Induction of Labor at Term: An Open-Label, Randomized Controlled Trial," PLoS One, 10(8), p. e0136856.

[12] Graham, M., Slocum, A., and Sanchez, R. M., 2007, "Teaching High School Students and College Freshmen Product Development by Deterministic Design With PREP," ASME J. Mech. Des., 129(7), pp. 677-681.

[13] Hanumara, N. C., Begg, N. D., Walsh, C. J., Custer, D., Gupta, R., Osborn, L. R., and Slocum, A. H., 2013, "Classroom to Clinic: Merging Education and Research to Efficiently Prototype Medical Devices," IEEE J. Transl. Eng. Health Med., 1, p. 4700107.

[14] Richmond, J. R., Morin, L., and Benjamin, A., 2002, "Extremely Preterm Vaginal Breech Delivery en Caul," Obstet. Gynecol., 99(6), pp. $1025-1030$.

[15] Hadlock, F. P., Deter, R. L., Harrist, R. B., and Park, S. K., 1984, "Estimating Fetal Age: Computer-Assisted Analysis of Multiple Fetal Growth Parameters," Radiology, 152(2), pp. 497-501.

[16] Myers, K., 2008, "An Investigation of the Structure-Function Relationship in Human Cervical Tissue," Ph.D. thesis, Massachusetts Institute of Technology, Cambridge, MA

[17] Paskaleva, A., 2007, "Biomechanics of Cervical Function in Pregnancy: Case of Cervical Insufficiency," Ph.D. thesis, Massachusetts Institute of Technology, Cambridge, MA.

[18] Myers, K., Paskaleva, A., House, M., and Socrate, S., 2008, "Mechanical and Biochemical Properties of Human Cervical Tissue," Acta Biomater., 4(1), pp. 104-116.

[19] Hee, L., Liao, D., Sandager, P., Gregersen, H., and Uldbjerg, N., 2014, "Cervical Stiffness Evaluated In Vivo by Endoflip in Pregnant Women," PLoS One, 9(3), p. e91121.

[20] Drunecký, T., Reidingerová, M., Plisová, M., Dudič, M., Gdovinová, D., and Stoy, V., 2015, "Experimental Comparison of Properties of Natural and Synthetic Osmotic Dilators," Arch. Gynecol. Obstet., 292(2), pp. 349-354.

[21] Allman, A. C. J., Genevier, E. S. G., Johnson, M. R., and Steer, P. J., 1996, "Head-to-Cervix Force: An Important Physiological Variable in Labour. 1. The Temporal Relation Between Head-to-Cervix Force and Intrauterine Pressure During Labour,” BJOG: An Int. J. Obstet. Gynaecol., 103(8), pp. 763-768.

[22] Timoshenko, S. P., and Gere, J. M., 1970, Theory of Elastic Stability, McGrawHill, New York.

[23] Weeks, G. E., 1967, "Buckling of a Pressurized Toroidal Ring Under Uniform External Loading," National Aeronautics and Space Administration, Washington, DC.

[24] Adolfo, R. P., and Soriano-Estrella, A. L., 2015, "Transcervical Foley Catheter Versus Laminaria: A Randomized Controlled Trial Comparing Efficacy and Safety in Facilitating Cervical Dilatation in Cases of Molar Pregnancies*," Philipp. J. Obstet. Gynecol., 39(4). 